\title{
Medical Costs and Healthcare Utilization among Cancer Decedents in the Last Year of Life in 2009
}

\author{
Inuk Hwang, $\mathrm{BS}^{1}$ \\ Dong Wook Shin, MD, DrPH, MBA ${ }^{2,3}$ \\ Kyoung Hee Kang, RN, MS 1 \\ Hyung Kook Yang, MD, MPH ${ }^{1}$ \\ So Young Kim, MD, PhD ${ }^{4}$ \\ Jong-Hyock Park, MD, MPH, PhD5,6
}

${ }^{1}$ National Cancer Control Institute, National Cancer Center, Goyang,

${ }^{2}$ Department of Family Medicine and

Health Promotion Center,

Seoul National University Hospital, Seoul,

${ }^{3}$ Cancer Survivorship Clinic, Seoul

National University Cancer Hospital, Seoul,

${ }^{4}$ Chungbuk Regional Cardiocerebrovascular Center,

Chungbuk National University Hospital,

Seoul, ${ }^{5}$ Graduate School of Health Science

Business Convergence,

Chungbuk National University, Cheongju,

${ }^{6}$ Department of Preventie Medicine,

Chungbuk National University

College of Medicine, Cheongju, Korea

Correspondence: Jong-Hyock Park, MD, MPH, PhD Department of Preventive Medicine,

Chungbuk National University

College of Medicine, 52 Naesudong-ro,

Seowon-gu, Cheongju 28644, Korea

Tel: 82-43-261-2873

Fax: 82-43-261-3459

E-mail: jonghyock@chungbuk.ac.kr

Co-correspondence: So Young Kim, MD, PhD

Chungbuk Regional Cardiocerebrovascular

Center, Chungbuk National University Hospital,

776 1sunhwan-ro, Seowon-gu,

Cheongju 28644, Korea

Tel: 82-43-261-3460

Fax: 82-43-266-6775

E-mail: letter.sykim@gmail.com

Received April 4, 2014

Accepted November 12, 2014

Published Online March 2, 2015

\section{Purpose}

The purpose of this study was to evaluate the cancer care cost during the last year of life of patients in Korea.

\section{Materials and Methods}

We studied the breakdown of spending on the components of cancer care. Cancer decedents in 2009 were identified from the Korean Central Cancer Registry and linked with the Korean National Health Insurance Claims Database. The final number of patients included in the study was 70,558 .

\section{Results}

In 2009, the average cancer care cost during the last year of life was US $\$ 15,720$. Patients under age 20 spent US $\$ 53,890$ while those 70 or over spent US $\$ 11,801$. Those with leukemia incurred the highest costs (US $\$ 43,219$ ) while bladder cancer patients spent the least (US $\$ 13,155)$. General costs, drugs other than analgesics, and test fees were relatively high (29.7\%, 23.8\%, and $20.7 \%$ of total medical costs, respectively). Analgesic drugs, rehabilitation, and psychotherapy were still relatively low $(4.3 \%, 0.7 \%$, and $0.1 \%$, respectively). Among the results of multiple regression analysis, few were notable. Age was found to be negatively related to cancer care costs while income level was positively associated. Those classified under distant Surveillance, Epidemiology, and End Results stages of cancer and higher comorbidity level also incurred higher cancer care costs.

\section{Conclusion}

Average cancer care costs varied significantly by patient characteristics. However, the study results suggest an underutilization of support services likely due to lack of alternative accommodations for terminal cancer patients. Further examination of utilization patterns of healthcare resources will help provide tailored evidence for policymakers in efforts to reduce the burdens of cancer care. 


\section{Introduction}

The burden of cancer and cancer care in developed countries is projected to rise due to both higher prevalence and advancing medical technology $[1,2]$. In Korea, cancer has been the leading cause of death since the year 2000 [3] and cancer incidence is expected to rise from 202,053 in 2010 to 270,809 in 2015 [4]. In other words the societal burden of cancer in Korea is substantial and increasing rapidly.

Cancer care costs vary by the clinical care phase, and a significant portion of the expenditure occurs in the last year of life, consistent with the intensity of care $[1,5,6]$. Chastek et al. [6] noted that terminal cancer patients spent US $\$ 74,212$ on average during their last 6 months of life, which is considerably higher than the average medicare beneficiary's expenditure of US $\$ 35,156$ in the last year of life [7] (both in 2009 US \$) and likely attributable to costly cancer treatments.

However, studies on end-of-life medical expenditures for cancer are relatively limited in Korea. Yi et al. [8] investigated cancer care costs by the site of cancer and survival time but did not fully explore other possible predictors of costs. The study by Choo et al. [9] focused on the effects of education level on cancer care cost but failed to examine other costdetermining factors, such as comorbidities and survival time. Choi et al. [10] compared cost differences between receiving hospice care and conventional care. However, it focused mainly on comparing medical expenses between a small patient group $(\mathrm{n}=529)$ who used hospice care and a comparison group $(n=1,908)$ who did not, and it was limited in terms of estimating cancer care costs in a representative sample. Lastly, none of these studies provided a breakdown of spending on the components of cancer care.

In the current study, we assess the cancer care cost in the last year of life by treatment component to examine healthcare utilization patterns of terminal cancer patients. In addition to variables that Yi et al. [8] and Choi et al. [10] found to affect the cancer care cost, we incorporated monthly insurance premium level, Surveillance, Epidemiology, and End Results (SEER) cancer stage, and Charlson comorbidty index level into our analysis. We examined each variable's effect on the cancer care cost, including income level, represented by the insurance premium level, which is known to cause inequalities in health care utilization by cancer patients in Korea [11]. Also, we observed few representative variables of a cancer patient's health care utilization pattern, including the frequency of diagnostic imaging that is non-negligible contributor to the overall cancer care cost [12]. To secure reliable data, we used the Korean National Health Insurance (KNHI) database. KNHI is a nationwide population-based database in which nearly all treatment-related information is available because medical providers are paid on a fee- for-service basis in Korea. Using this unique data set, we estimated cancer care costs during the last year of life according to patient characteristics and the components of cancer care treatment, which, in turn, enabled us to identify healthcare utilization patterns of the terminal cancer patients.

\section{Materials and Methods}

\section{Data sources and study patient identification}

The two major data sources used were the Korean Central Cancer Registry (KCCR) and the KNHI claims database. $\mathrm{KCCR}$ is a nationwide cancer registry program sponsored by the government in which more than 180 hospitals participate. The completeness of registration for 2009 was estimated to be $97.2 \%$ of cancer cases [13]. For each patient, the KCCR included information on gender, age at diagnosis, date of diagnosis, cancer site, histology, and the SEER summary stage. Among 878,834 cancer cases in the KCCR in 2009, 72,563 cancer decedents were selected. Patients with incomplete death information $(n=1,836)$ and those with no medical claims history $(\mathrm{n}=139)$ were excluded, leaving 70,558 patients in the study (Fig. 1). The study subjects were matched with the KNHI claims. KNHI scheme was launched by the government in 1989 as a mandatory universal health insurance program for the whole population, and the KNHI database contains claims data for medical services in Korea. It includes information on patient gender, age, health insurance premiums, residential area, comorbid diseases, specific surgical procedures, and medical expenditures [14].

Cancer patients were classified by tumor type for those with a cancer diagnosis of stomach, lung, liver, colon and rectum, breast, cervix uteri, thyroid, gallbladder, pancreas, bladder, prostate, kidney, esophagus, ovary, leukemia, oral cavity, larynx, melanoma of skin, brain and central nervous system, non-Hodgkin's disease, Hodgkin's disease, multiple myeloma, testis, corpus uteri, nasopharynx, and other pharynx. All other tumor types were grouped as "others."

This study was approved by the Korean National Cancer Center Institutional Review Board. The requirement for informed consent was waived, because the study was based on routinely collected administrative or registry data.

\section{Variables and outcomes}

The following patient characteristics were included: gender, age, insurance premium level, residential area, medical utilization, SEER summary stage, Charlson comorbidity 


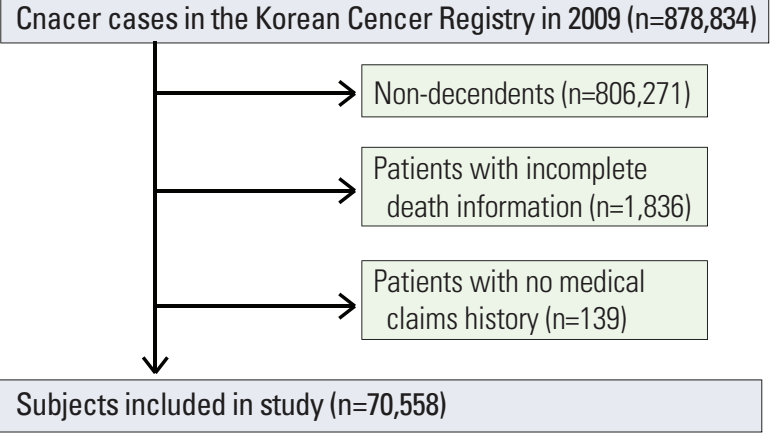

Fig. 1. Disposition of study subjects.

index, survival time, and site of cancer.

Patient age at death was divided into five categories: $<20$, 20-49, 50-59, 60-69, and $\geq 70$ years. Based on income levels, we identified individuals in the insurance premium categories of below poverty line (lowest), I, II, III, IV, and V (highest) to create income groups with approximately equal numbers of subjects. KNHI contribution was used as a proxy measure for actual household income because it is calculated based on the income, property, and private auto taxes for each household [11]. Patients were grouped into three residential area categories of metropolitan, urban, and rural, according to Korean ZIP code. We also used the SEER summary stage information within the KCCR data which categorizes how far a cancer has spread from its origin. The four categories of Summary Staging information available in the KCCR data are localized, regional, distant, and unknown. Localized cancer refers to a malignancy limited to the organ of origin. Regional stage refers to a state where a tumor extends beyond the limits of the organ of origin. Distant stage includes tumors that have spread to areas of the body distant from the primary tumor. Lastly, cases for which sufficient evidence is not available to adequately assign stage are categorized under unknown [15]. The Charlson comorbidity index, which is a single index of comorbidity burden developed to assess the relative risk of a patient's comorbid conditions for determining patient outcome after a critical illness, was used to group patients into four categories according to the index score: $0,1-2,3-4$, and $\geq 5$ (the most severe). Survival time was defined as the period from the date of cancer diagnosis to that of death or end of follow-up. The date of diagnosis was obtained from the KCCR. For cases in which patients were diagnosed with multiple cancers, we classified patients according to the last diagnosis.

We classified medical institutions as clinics and small hospitals, general hospitals, tertiary hospitals, or tertiary referral hospitals. Clinics are small, private medical facilities dedicated to outpatient services and equipped with up to 30 beds. Small hospitals are equipped with more than 30 beds. General hospitals provide specialist care and are equipped with more than 100 beds. Tertiary hospitals are general hospitals that are usually affiliated with universities, provide a certain quality of care, perform research, and act as teaching institutions. Tertiary referral hospitals are those that provide outstanding medical care [11].

Patterns of healthcare utilization were also assessed. Namely the mean number of inpatient days/visits, outpatient days/visits, and emergency department visits and the use of computed tomography (CT), positron emission tomography (PET), and magnetic resonance imaging (MRI) were examined. In addition, the components of cancer care costs were examined as follows: identifying costs associated with radiotherapy, chemotherapy, surgeries (e.g., resection), general tests (e.g., lab tests and X-rays), diagnostic imaging (e.g., CT, PET, MRI, angiography, nuclear medicine imaging, and bone densitometry), analgesic drugs (e.g., morphine and nonsteroidal anti-inflammatory drugs), drugs other than analgesics, general costs (e.g., fees for physician consultations, inpatient admission, meals, basic treatment such as incisions and dressings), rehabilitation, and psychotherapy.

The last year of life was defined as the final 12 months of life, and the mean medical cost in the last year of life was calculated by combining the medical claims for each individual [8]. All cost measures were computed as combined insurer and co-payment amounts for each claim and were adjusted to 2009 US $\$$ using an annual average exchange rate of 1,164.5 Korean won in 2009.

\section{Statistical analyses}

Descriptive analyses were performed for the categories of decedent gender, age, insurance premium level, residential area, SEER stage, Charlson comorbidity index, survival time, emergency department use, site of cancer, and medical utilization. Multiple linear regression analyses were performed to estimate the association between mean cancer care cost during the last year of life and decedent's demographic and clinical characteristics. To correct for the skewed distribution of the medical cost data, we used the log-transformed mean cancer care cost as the outcome variable. All analyses were performed using SAS software ver. 9.2 (SAS Institute Inc., Cary, NC). Two-sided p-values of $\leq 0.05$ were considered to indicate statistical significance. 
Table 1. Demographic and clinical characteristics of patients

\begin{tabular}{|c|c|c|}
\hline Characteristic & No. $(\%)$ & Mean \pm SD (US \$) \\
\hline \multicolumn{3}{|l|}{ Sex } \\
\hline Male & $44,884(63.6)$ & $15,668 \pm 14,045$ \\
\hline Female & $25,674(36.4)$ & $15,810 \pm 14,810$ \\
\hline \multicolumn{3}{|l|}{ Age (yr) } \\
\hline$<20$ & $323(0.5)$ & $53,890 \pm 39,099$ \\
\hline $20-49$ & $6,389(9.1)$ & $23,743 \pm 21,218$ \\
\hline $50-59$ & $10,305(14.6)$ & $20,240 \pm 16,413$ \\
\hline $60-69$ & $16,801(23.8)$ & $17,730 \pm 13,238$ \\
\hline$\geq 70$ & $36,740(52.1)$ & $11,801 \pm 9,998$ \\
\hline Mean \pm SD & $68.0 \pm 13.4$ & - \\
\hline \multicolumn{3}{|l|}{ Insurance premium, quintiles } \\
\hline Below poverty line (lowest) & $8,680(12.3)$ & $15,094 \pm 12,851$ \\
\hline I & $9,343(13.2)$ & $15,054 \pm 14,625$ \\
\hline II & 8,327 (11.8) & $15,590 \pm 13,738$ \\
\hline III & $10,452(14.8)$ & $16,484 \pm 14,826$ \\
\hline IV & $12,889(18.3)$ & $16,256 \pm 15,097$ \\
\hline V (highest) & $20,002(28.4)$ & $15,654 \pm 14,298$ \\
\hline \multicolumn{3}{|l|}{ Residential area } \\
\hline Metropolitan & $37,300(52.9)$ & $16,702 \pm 15,231$ \\
\hline Urban & $20,947(29.7)$ & $15,166 \pm 13,714$ \\
\hline Rural & $12,298(17.4)$ & $13,694 \pm 12,094$ \\
\hline \multicolumn{3}{|l|}{ SEER stage } \\
\hline Localized & $12,283(17.4)$ & $15,134 \pm 13,239$ \\
\hline Regional & $16,067(22.8)$ & $15,668 \pm 11,696$ \\
\hline Distant & $22,549(32.0)$ & $17,911 \pm 17,224$ \\
\hline Unknown & 7,853 (11.1) & $13,289 \pm 12,076$ \\
\hline \multicolumn{3}{|l|}{ Charlson comorbidity index } \\
\hline 0 & $32,981(46.7)$ & $15,771 \pm 15,298$ \\
\hline $1-2$ & $25,747(36.5)$ & $15,434 \pm 13,550$ \\
\hline $3-4$ & $8,796(12.5)$ & $15,759 \pm 12,995$ \\
\hline$\geq 5$ & $3,034(4.3)$ & $17,471 \pm 13,382$ \\
\hline \multicolumn{3}{|l|}{ Survival time (yr) } \\
\hline$<1$ & 37,007 & $14,777 \pm 13,524$ \\
\hline $1-2$ & 13,111 & $18,864 \pm 16,371$ \\
\hline $2-5$ & 13,808 & $16,135 \pm 14,197$ \\
\hline$>5$ & 6,632 & $13,897 \pm 13,636$ \\
\hline Mean \pm SD & $1.8 \pm 2.1$ & - \\
\hline \multicolumn{3}{|l|}{ Emergency department use } \\
\hline No & $45,810(64.9)$ & $13,345 \pm 12,239$ \\
\hline Yes & $24,748(35.1)$ & $20,115 \pm 16,682$ \\
\hline \multicolumn{3}{|l|}{ Site of cancer } \\
\hline Stomach & $11,044(15.7)$ & $13,260 \pm 10,532$ \\
\hline Lung & $14,070(19.9)$ & $15.596 \pm 11,329$ \\
\hline Liver & $11,290(16.6)$ & $13,584 \pm 11.296$ \\
\hline Colon and rectum & 7,671 (10.9) & $14,224 \pm 11,287$ \\
\hline Breast & $1,798(2.6)$ & $17,650 \pm 11,918$ \\
\hline Cervix uteri & $981(1.4)$ & $16,587 \pm 13,424$ \\
\hline Thyroid & $638(0.9)$ & $14,858 \pm 14,723$ \\
\hline Gallbladder & $3,171(4.5)$ & $13,363 \pm 9,621$ \\
\hline Pancreas & $3,693(5.2)$ & $13,467 \pm 9,034$ \\
\hline Bladder & $1,221(1.7)$ & $13,155 \pm 11,366$ \\
\hline
\end{tabular}


Table 1. Continued

\begin{tabular}{|c|c|c|}
\hline Characteristic & No. $(\%)$ & Mean \pm SD (US \$) \\
\hline Prostate & $1,558(2.2)$ & $13,252 \pm 10,758$ \\
\hline Kidney & $863(1.2)$ & $17,297 \pm 13,288$ \\
\hline Esophagus & $1,436(2.0)$ & $16,587 \pm 12,928$ \\
\hline Ovary & $780(1.1)$ & $19,250 \pm 13,766$ \\
\hline Leukemia & $1,407(2.0)$ & $43,219 \pm 38,636$ \\
\hline Oral cavity & $495(0.7)$ & $16,198 \pm 12,811$ \\
\hline Larynx & $418(0.6)$ & $13,908 \pm 13,072$ \\
\hline Melanoma of skin & $204(0.3)$ & $15,069 \pm 11,494$ \\
\hline Brain and central nervous system & $921(1.3)$ & $24,232 \pm 18,029$ \\
\hline Non-Hodgkin's disease & $1,364(1.9)$ & $28,012 \pm 25,440$ \\
\hline Hodgkin's disease & $51(0.1)$ & $23,918 \pm 20,452$ \\
\hline Multiple myeloma & $679(1.0)$ & $26,491 \pm 21,125$ \\
\hline Testis & $17(0.0)$ & $25,714 \pm 14,283$ \\
\hline Corpus uteri & $255(0.4)$ & $17,675 \pm 13,765$ \\
\hline Nasopharynx & $187(0.3)$ & $17,573 \pm 13,185$ \\
\hline Other pharynx & $399(0.6)$ & $18,076 \pm 14,941$ \\
\hline Others & $3,947(5.6)$ & $17,183 \pm 18,797$ \\
\hline Total & $70,558(100)$ & \\
\hline \multicolumn{3}{|l|}{ Phase of care } \\
\hline Entire cancer experience & - & $23,379 \pm 22,273$ \\
\hline Last year of life & - & $15,720 \pm 14,328$ \\
\hline \multicolumn{3}{|l|}{ Healthcare utilization (mean \pm SD) } \\
\hline Inpatient days / visits & $71 \pm 70 / 6 \pm 5$ & - \\
\hline Outpatient days/visits & $40 \pm 34 / 63.6 \pm 58.7$ & - \\
\hline Emergency room visits & $0.7 \pm 1.3$ & - \\
\hline CT use & $4.0 \pm 3.1$ & - \\
\hline PET/CT use & $0.6 \pm 0.9$ & - \\
\hline MRI use & $0.8 \pm 1.6$ & - \\
\hline
\end{tabular}

SD, standard deviation; SEER, Surveillance, Epidemiology, and End Results; CT, computed tomography; PET, positron emission tomography; MRI, magnetic resonance imaging.

\section{Results}

\section{Characteristics of the study population}

Patient demographic and clinical characteristics are summarized in Table 1. Individuals 70 years of age or older accounted for $52.1 \%$ of the patients, and the mean age was 68.0 years (standard deviation [SD], 13.4 years). There were 8,680 patients $(12.3 \%)$ below poverty line, and the largest percentage $(28.4 \%)$ was in the highest income class (V). Over $80 \%$ of patients lived in a metropolitan or urban area. Because the study population consisted of decedents, most $(n=22,549,32.0 \%)$ were classified under the distant SEER summary stage. However, most of the patients $(n=58,728$, $83.2 \%$ ) scored $\leq 2$ on the Charlson comorbidity index, suggesting a relatively low level of comorbidity. Of the subjects, $35.1 \%$ used the emergency department at least once during the last year of life. Lung cancer was the most frequent type of cancer $(\mathrm{n}=14,070,19.9 \%)$, followed by liver $(\mathrm{n}=11,290,16.0 \%)$ and stomach $(\mathrm{n}=11,044,15.7 \%)$ cancers. The mean survival time was 1.8 years (SD, 2.1 years).

\section{Cancer care cost and healthcare utilization}

Cancer care costs and healthcare utilization of patients in the last year of life are presented in Table 1. We observed considerable variation in cancer care costs. Patients younger than 20 years spent the most while those 70 years or older spent the least. The effects of residential area were also examined, revealing that patients living in metropolitan areas had higher costs than those in urban and rural areas. 
A
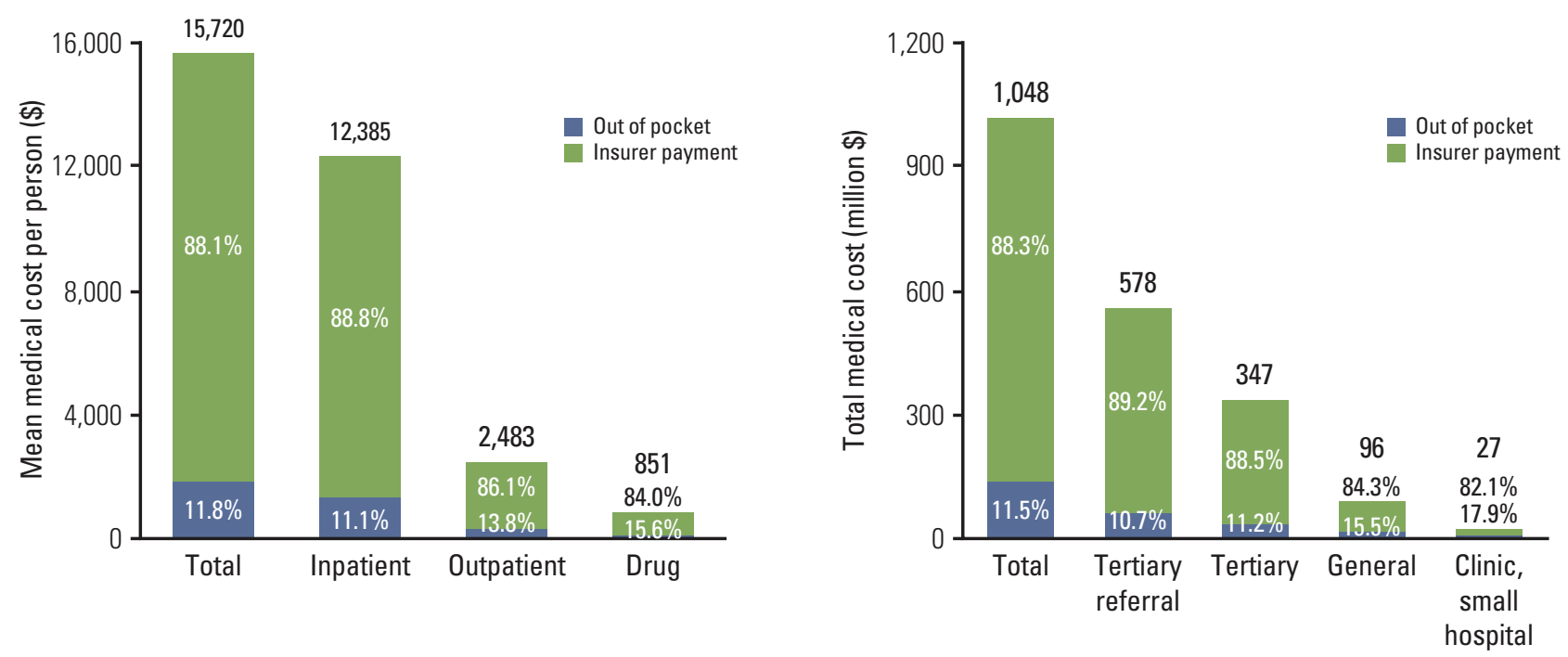

Fig. 2. Distribution of cancer care costs by types of services and hospitals. (A) On average, a cancer decedent spent US $\$ 15,720$ in the last year of life, of which majority was associated with inpatient services (US $\$ 12,385$ ). (B) Nearly $90 \%$ of the overall medical expenditure occurred in the tertiary referral (US \$578 million) and tertiary hospitals (US \$347 million), suggesting excessive use of upper level hospitals.

Over half of the decedents had survival times of less than 1 year, and those surviving 1 to 2 years incurred the highest costs (US $\$ 18,864$ ), whereas patients surviving more than 5 years incurred the lowest costs (US $\$ 13,897$ ). Patients with leukemia incurred the highest mean medical costs (US $\$ 43,219$ ), followed by non-Hodgkin's disease (US $\$ 28,012$ ), multiple myeloma (US \$26,491), testicular cancer (US \$25,714), and brain and central nervous system cancers (US \$24,232). On average, patients were hospitalized for 71 days, spent 40 days equivalent time using outpatient services, visited the emergency department 0.7 times, and underwent CT, PET, and MRI 4, 0.6, and 0.8 times, respectively (Table 1 ).

\section{Cancer care costs according to the category of service and type of hospitals}

The distribution of cancer care costs by the category of service is presented in Fig. 2A. The majority was attributable to inpatient costs (US $\$ 12,385,78.8 \%$ ), followed by outpatient costs (US $\$ 2,483,15.8 \%$ ) and drug costs (US $\$ 851,5.4 \%$ ). Fig. $2 \mathrm{~B}$ presents the distribution of cancer care costs by the type of medical institution. Among total cancer care costs (US $\$ 1,048$ million), those at tertiary referral hospitals were the highest (\$578 million, 55.1\%), followed by tertiary hospitals (\$347 million, 29.3\%), general hospitals (\$96 million, 9.2\%), and clinics (\$27 million, 2.6\%), indicating a higher rate of larger hospital use by terminal cancer patients.

\section{Cancer care costs according to the time before death}

Cancer care costs evaluated according to the time before death are shown in Fig. 3. Costs increased steadily as the patient approached death, from $\$ 644$ in the 12th month to $\$ 2,480$ in the last month before death. The highest medical costs occurred 2 months prior to death $(\$ 2,665,17.0 \%)$, and $69.5 \%$ of the total medical expenditure during the last year of life was spent in the last 6 months.

\section{Cancer care costs according to treatment type}

Fig. 4 presents cancer care costs by the type of treatment component and survival time. On average, general costs were the highest $(29.7 \%)$, followed by other drugs (23.8\%), general tests $(14.3 \%)$, chemotherapy $(11.6 \%)$, imaging tests $(6.4 \%)$, surgery $(4.7 \%)$, analgesia $(4.3 \%)$, radiotherapy $(2.9 \%)$, rehabilitation $(0.7 \%)$, and psychotherapy $(0.1 \%)$. Costs associated with chemotherapy, surgery, radiotherapy, and imaging tests were higher in patients with shorter survival time, although the differences were small. And costs associated with psychotherapy and rehabilitation, which are supportive care services, were very low regardless of patients' survival time. 


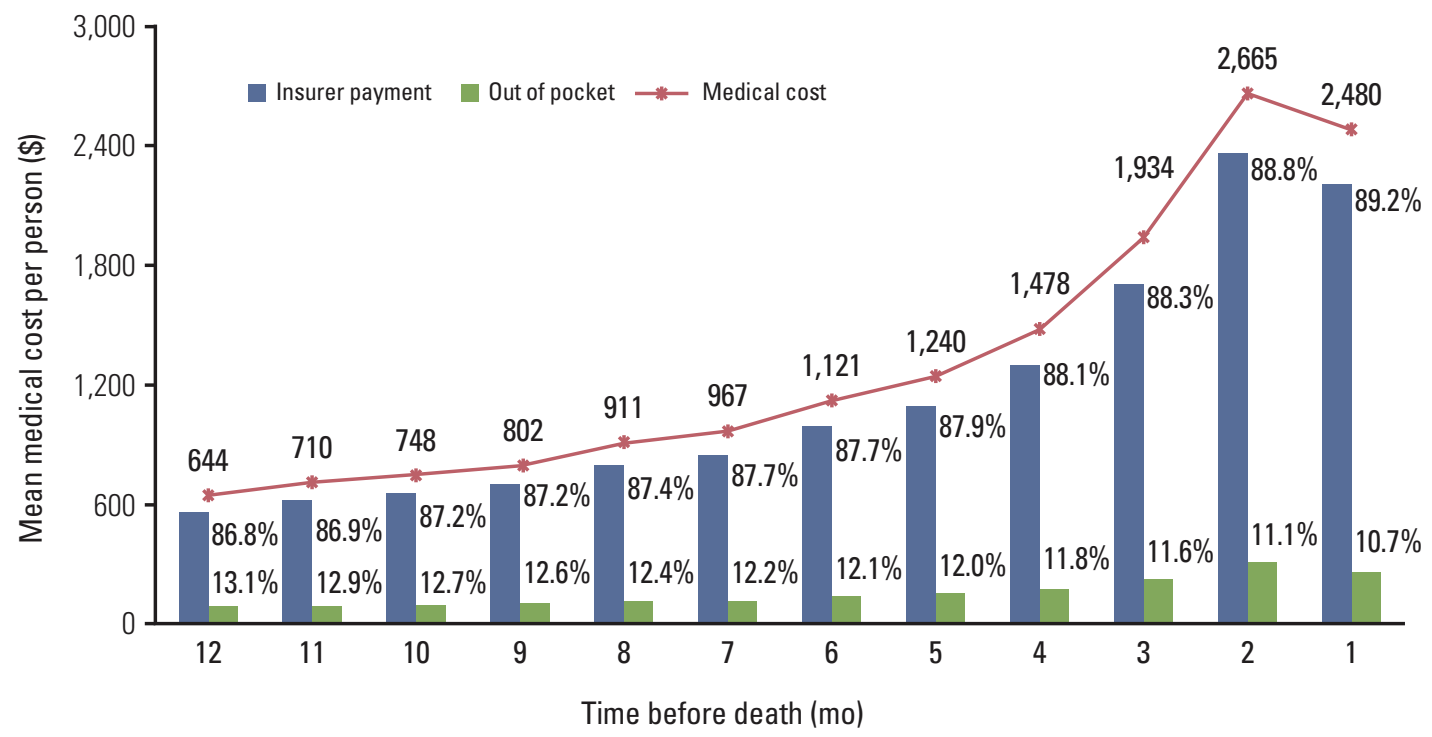

Fig. 3. Expenditures per month during the last year of life.

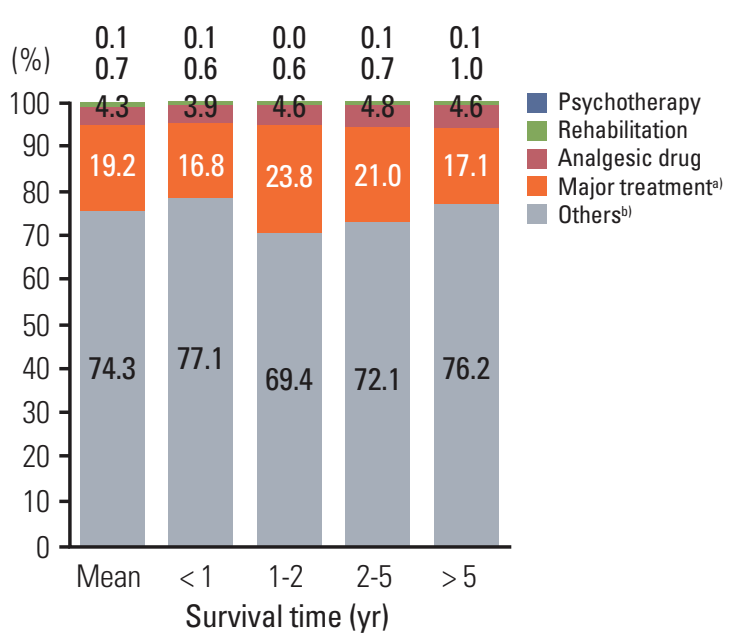

Fig. 4. Medical costs by survival time and treatment type. a)Major treatment: radiotherapy, surgery, chemotherapy, b)Others: imaging test, general test, other drug, general costs.

\section{Predictors of cancer care costs}

The predictors of cancer care costs are presented in Table 2. In our study population, older age was negatively associated with cancer care costs. Higher income level, indicated by the insurance premium, was positively associated with cancer care costs, although insurance premium levels III and IV showed no statistically significant association. Compared with patients living in metropolitan areas, those in urban and rural areas were more likely to have lower costs. As would be expected, having more progressed cancer and higher comorbidity scores were also associated with higher mean medical costs. Survival time was positively associated with cancer care costs, although surviving more than 5 years was associated with lower costs, albeit not statistically significantly so. Finally, using the emergency department in the last year of life resulted in relatively high costs.

\section{Discussion}

Estimating disease-specific medical costs is of longstanding interest in the fields of health policy and health economics [16]. At the macro-level, such estimates can provide references for budget planning [17] and are necessary for monitoring the flow of national health expenditures. The estimates of phase-specific medical costs presented in this study may be used to evaluate the cost-effectiveness of cancer control policies.

In our study, the average medical cost varied by the decedent's characteristics. Those younger than 20 years had the highest mean medical costs, consistent with previous results [8] showing that lower age was associated with higher cancer care costs. This is associated with the tendency of younger cancer patients to seek more aggressive surgical care and adjuvant treatment [18] compared with older patients. Also, medical costs increased with insurance premium levels, 
Table 2. Predictors of cancer care costs during the last year of life

\begin{tabular}{|c|c|c|c|c|}
\hline \multirow{2}{*}{ Variable } & \multicolumn{2}{|c|}{ Unadjusted } & \multicolumn{2}{|c|}{ Adjusted } \\
\hline & Coefficient & p-value & Coefficient & p-value \\
\hline \multicolumn{5}{|l|}{ Sex } \\
\hline \multicolumn{5}{|l|}{ Male } \\
\hline Female & -0.0071 & 0.340 & -0.0469 & $<0.001$ \\
\hline \multicolumn{5}{|l|}{ Age (yr) } \\
\hline \multicolumn{5}{|l|}{$<20$} \\
\hline $20-49$ & -0.8314 & $<0.001$ & -0.5149 & $<0.001$ \\
\hline $50-59$ & -0.9518 & $<0.001$ & -0.5747 & $<0.001$ \\
\hline $60-69$ & -1.0741 & $<0.001$ & -0.6089 & $<0.001$ \\
\hline$\geq 70$ & -1.5490 & $<0.001$ & -0.7465 & $<0.001$ \\
\hline \multicolumn{5}{|c|}{ Insurance premium, quintile } \\
\hline \multicolumn{5}{|c|}{ Below poverty line (lowest) } \\
\hline I & -0.0666 & $<0.001$ & 0.0520 & $<0.001$ \\
\hline II & -0.0218 & 0.132 & 0.0702 & $<0.001$ \\
\hline III & 0.0422 & 0.002 & 0.0851 & $<0.001$ \\
\hline IV & 0.0232 & 0.077 & 0.0801 & $<0.001$ \\
\hline V (highest) & 0.0018 & 0.881 & 0.0983 & $<0.001$ \\
\hline \multicolumn{5}{|l|}{ Residential area } \\
\hline \multicolumn{5}{|l|}{ Metropolitan } \\
\hline Urban & -0.1032 & $<0.001$ & -0.0458 & $<0.001$ \\
\hline Rural & -0.2027 & $<0.001$ & -0.0787 & $<0.001$ \\
\hline \multicolumn{5}{|l|}{ SEER cancer stage } \\
\hline \multicolumn{5}{|l|}{ Localized } \\
\hline Regional & 0.0951 & $<0.001$ & 0.0002 & 0.978 \\
\hline Distant & 0.1879 & $<0.001$ & 0.0452 & $<0.001$ \\
\hline Unknown & -0.2154 & $<0.001$ & -0.0048 & 0.735 \\
\hline \multicolumn{5}{|c|}{ Charlson comorbidity index } \\
\hline \multicolumn{5}{|c|}{0} \\
\hline $1-2$ & 0.0751 & $<0.001$ & 0.0207 & $<0.001$ \\
\hline $3-4$ & 0.1306 & $<0.001$ & 0.0486 & $<0.001$ \\
\hline$\geq 5$ & 0.2632 & $<0.001$ & 0.1129 & $<0.001$ \\
\hline \multicolumn{5}{|l|}{ Survival time (yr) } \\
\hline \multicolumn{5}{|l|}{$<1$} \\
\hline $1-2$ & 0.2192 & $<0.001$ & 0.0958 & $<0.001$ \\
\hline $2-5$ & 0.0300 & 0.001 & 0.0783 & $<0.001$ \\
\hline$\geq 5$ & -0.2480 & $<0.001$ & 0.0924 & $<0.001$ \\
\hline \multicolumn{5}{|c|}{ Emergency department use } \\
\hline \multicolumn{5}{|c|}{ No } \\
\hline Yes & 0.5355 & $<0.001$ & 0.1451 & $<0.001$ \\
\hline \multicolumn{5}{|c|}{ Healthcare utilization } \\
\hline Inpatient days & 0.5754 & $<0.001$ & 0.4843 & $<0.001$ \\
\hline Outpatient days & 0.2698 & $<0.001$ & 0.1451 & $<0.001$ \\
\hline CT use & 0.1705 & $<0.001$ & 0.0637 & $<0.001$ \\
\hline PET/CT use & 0.2960 & $<0.001$ & 0.0595 & $<0.001$ \\
\hline MRI use & 0.1853 & $<0.001$ & 0.0322 & $<0.001$ \\
\hline Adjusted-R & & & 0.7202 & \\
\hline
\end{tabular}

Multivariable adjusted models were adjusted for gender, age, insurance premium, residential area, insurance type, level of disability, Charlson score, Surveillance, Epidemiology, and End Results (SEER) stage, survival time, and cancer type. CT, computed tomography; PET, positron emission tomography; MRI, magnetic resonance imaging. 
accounted for by high-income patients using inpatient and outpatient care more frequently than those from lower income groups [11]. However, the medical expenditures of insurance premium level groups I and II were statistically significantly lower than those below poverty line, indicating the underutilization of healthcare services by the near-poor, because income level and healthcare use are generally positively associated. It may also indicate that the near-poor have insufficient access to healthcare services in Korea. Finally, patient populations in metropolitan areas spent more than those in rural areas, consistent with the previous study by Kim and Park [19], who noted higher cancer care costs in urban populations.

Examining the distribution of expenses by the type of service revealed a disproportionate result, in that nearly $80 \%$ of medical costs were attributable to inpatient care. Although Chastek et al. [6] reported that inpatient costs accounted for the largest proportion of end-of-life costs in the United States $(55 \%)$, we found that this proportion was much higher $(78.7 \%)$ in the Korean population. It is believed that the relative lack of alternative accommodation for terminal cancer patients (such as home care) in Korea contributed to a higher rate of hospitalization.

Additional research is warranted to assess hospital utilization patterns in terminal cancer patients in Korea. In our analysis, $35.1 \%$ of decedents had visited the emergency department at least once in the last year of life. The study by Keam et al. [20] reported a similar rate of emergency department use in Korea (33.6\%) which is noted to be much higher than that reported in the United States $(9.2 \%$ in 1996) and suggesting inadequate functioning of regional medical facilities in Korea. It is also noted that establishing a continuing cancer care system based on the family physician setting [20] may lower the emergency room visit rate.

The distribution of expenses according to the type of hospital was also disproportionate. Over half of total medical costs incurred at tertiary referral hospitals, which are preferred by patients with serious diseases such as cancer [9]. However, this may represent inappropriate hospital use and unnecessary medical costs, simply because most terminal cancer patients do not require the specialized care provided by tertiary referral hospitals.

Our study showed that $11.6 \%$ and $2.99 \%$ of medical costs during the last 6 months of life were spent on chemotherapy and radiotherapy, respectively. This is consistent with the report by Chastek et al. [6], who noted that terminal cancer patients spent $10 \%$ and $5 \%$ of their medical costs during the last 6 months on chemotherapy and radiotherapy, respectively. The tendency to administer aggressive chemotherapy until the end of life in Korea was noted by Keam et al. [20], who showed that as many as $94.6 \%$ of patients received chemotherapy during the last 6 months of life, much higher than that reported in the United States (33\%). The cost of chemotherapy was highest in patients surviving 1 to 2 years. Previous studies [21,22] noted that rehabilitation and psychotherapy can make meaningful contributions, even to terminal cancer patients. Specifically, Yoshioka [22] noted that almost all study subjects experienced at least some relief from certain types of discomfort, such as pain, dyspnea, and leg edema. According to a report published by the Irish Cancer Society [23], 9\% and 6\% of participants surveyed incurred costs for physiotherapy and counseling, respectively. However, patients in the current study spent $1 \%$ and $0 \%$ of their medical costs on rehabilitation and psychotherapy, respectively, which may indicate underutilization of such services in Korea.

In our study, on average, patients underwent $4 \mathrm{CT}, 0.6$ PET, and 0.8 MRI scans in the last year of life, representing $6.4 \%$ of mean cancer care costs. In comparison, Dinan et al. [12] noted that patients with lymphoma underwent the highest number of imaging procedures, and an average lymphoma patient in 2006 had six CT scans, one PET scan, and one MRI within 2 years of diagnosis. Moreover, using the cost data of this study, Yang and Czernin [24] estimated that imaging expenditures in 2006 accounted for $4.6 \%$ of overall medicare cancer care costs. Considering that our study subjects were terminal cancer patients, who require relatively less diagnostic imaging, and that the hospital utilization pattern was examined over a 1-year period, the use of diagnostic imaging in Korea may be excessive; this should be investigated further.

There are several limitations to our study. First, we used an administrative claims database, which contains the cost of billed services only, not including costs associated with loss of patient/caregiver productivity/wages, travel to/from treatment, over-the-counter medications, and other cancerrelated expenses [6]. However, the results of this study were consistent with previous studies that used KNHI claims data, demonstrating high costs during the last year of life for many conditions, including cancer. Second, rather than computing expenditures specifically related to cancer care, this study estimated all medical expenditures incurred by patients dying of cancer. However, a previous study [25] noted that most expenditures incurred by cancer patients during the last year of life were attributable to cancer care. Thus, it is likely that this was true in our study as well. Third, in this study we accounted for expenditures for services that are covered by KNHI only. Therefore, cost estimates of this study do not reflect additional healthcare costs that are not covered by KNHI or costs to society due to premature death and lost productivity. Despite these limitations, our study was strong in that the study population represented a large segment (97\%) of cancer mortality cases in Korea in 2009. 


\section{Conclusion}

To our knowledge, this is the first report to document the breakdown of spending on the components of cancer care during the last year of life in Korea. Our study results demonstrate that cancer care costs during this time are a significant burden to patients and illustrate the need for further research on costs associated with the components of cancer care. Examining possible overutilization and underutilization of healthcare resources will help provide tailored evidence for policymakers in efforts to reduce the burdens of cancer care.

\section{Conflicts of Interest}

Conflict of interest relevant to this article was not reported.

\section{Acknowledgments}

This work was supported by grants from the National R\&D Program for Cancer Control and Chungbuk National University in 2014.

\section{References}

1. Yabroff KR, Warren JL, Brown ML. Costs of cancer care in the USA: adescriptive review. Nat Clin Pract Oncol. 2007;4: 643-56.

2. Mariotto AB, Yabroff KR, Shao Y, Feuer EJ, Brown ML. Projections of the cost of cancer care in the United States: 20102020. J Natl Cancer Inst. 2011;103:117-28.

3. Statistics Korea. Annual report on the cause of death statistics. Daejeon: Korea National Statistical Office; 2007.

4. National Cancer Center, Ministry of Health and Welfare. Cancer facts and figures 2013 in the Republic of Korea. Goyang: National Cancer Center; 2013.

5. Yabroff KR, Lund J, Kepka D, Mariotto A. Economic burden of cancer in the United States: estimates, projections, and future research. Cancer Epidemiol Biomarkers Prev. 2011;20: 2006-14.

6. Chastek B, Harley C, Kallich J, Newcomer L, Paoli CJ, Teitelbaum AH. Healthcare costs for patients with cancer at the end of life. J Oncol Pract. 2012;8:75s-80s.

7. Hogan C, Lunney J, Gabel J, Lynn J. Medicare beneficiaries' costs of care in the last year of life. Health Aff (Millwood). 2001;20:188-95.

8. Yi JJ, Yoo WK, Kim SY, Kim KK, Yi SW. Medical expenses by site of cancer and survival time among cancer patients in the last one year of life. J Prev Med Public Health. 2005;38:9-15.

9. Choo SY, Lee SY, Kim CW, Kim SY, Yoon TH, Shin HR, et al. Educational differences in health care utilization in the last year of life among South Korean cancer patients. J Prev Med Public Health. 2007;40:36-44.

10. Choi KS, You CH, Lee KH, Kim CY, Heo DS, Yun YH. Comparison of medical care cost between hospice care and conventional care in the last year of life. Korean J Health Policy Adm. 2005;15:1-15.

11. Yoon TH, Lee SY, Kim CW, Kim SY, Jeong BG, Park HK. In equalities in medical care utilization by South Korean cancer patients according to income: a retrospective cohort study. Int J Health Serv. 2011;41:51-66.
12. Dinan MA, Curtis LH, Hammill BG, Patz EF Jr, Abernethy AP, Shea AM, et al. Changes in the use and costs of diagnostic imaging among Medicare beneficiaries with cancer, 1999-2006. JAMA. 2010;303:1625-31.

13. Jung KW, Park S, Kong HJ, Won YJ, Lee JY, Seo HG, et al. Cancer statistics in Korea: incidence, mortality, survival, and prevalence in 2009. Cancer Res Treat. 2012;44:11-24.

14. Kim SY, Park JH, Kim SG, Woo HK, Park JH, Kim Y, et al. Disparities in utilization of high-volume hospitals for cancer surgery: results of a Korean population-based study. Ann Surg Oncol. 2010;17:2806-15.

15. National Cancer Insitute. Review: summary staging [Internet]. Bethesda, MD: National Cancer Insitute; 2014 [cited 2014 Feb 2]. Available from: http://training.seer.cancer.gov/ss2k/staging/ review.html.

16. Brown ML, Lipscomb J, Snyder C. The burden of illness of cancer: economic costand quality of life. Annu Rev Public Health. 2001;22:91-113.

17. Yabroff KR, Mariotto AB, Feuer E, Brown ML. Projections of the costs associated with colorectal cancer care in the United States, 2000-2020. Health Econ. 2008;17:947-59.

18. Athas WF, Adams-Cameron M, Hunt WC, Amir-Fazli A, Key $\mathrm{CR}$. Travel distance to radiation therapy and receipt of radiotherapy following breast-conserving surgery. J Natl Cancer Inst. 2000;92:269-71.

19. Kim SG, Park WS. Out-of-pocket health expenditures among adult Koreans with cancer. Korean J Med. 2006;70:61-8.

20. Keam B, Oh DY, Lee SH, Kim DW, Kim MR, Im SA, et al. Aggressiveness of cancer-care near the end-of-life in Korea. Jpn J Clin Oncol. 2008;38:381-6.

21. Fawzy FI. Psychosocial interventions for patients with cancer: what works and what doesn't. Eur J Cancer. 1999;35:1559-64.

22. Yoshioka H. Rehabilitation for the terminal cancer patient. Am J Phys Med Rehabil. 1994;73:199-206.

23. Sharp L, Timmons A. The financial impact of a cancer diagnosis. Cork, IR: Irish Cancer Society; 2010. 
24. Yang Y, Czernin J. Contribution of imaging to cancer care costs. J Nucl Med. 2011;52 Suppl 2:86S-92S.

25. Brown ML, Riley GF, Schussler N, Etzioni R. Estimating health care costs related to cancer treatment from SEER-Medicare data. Med Care. 2002;40(8 Suppl):IV-104-17. 